\title{
Significato clinico della cinetica dell'urea nel paziente in dialisi
}

\author{
G. Panzetta
}

\author{
Servizio di Nefrologia e Dialisi, Ospedale Maggiore, Trieste
}

\section{La tossicità uremica}

ei pazienti con insufficienza renale cronica il metabolismo delle proteine ha un ruolo fondamentale nel determinare la produzione delle tossine uremiche, nel modulare l'omeostasi degli elettroliti e nel mantenere un appropriato stato di nutrizione. Infatti, adeguando l'introito proteico al grado di insufficienza renale è possibile mantenere molti pazienti uremici senza sintomi eccessivi.

L'identificazione dei fattori responsabili della tossicità uremica è stato oggetto di grande dibattito, tuttavia la definizione di questi fattori rimane tuttora incerta. La maggior parte delle sostanze tossiche deriva, almeno potenzialmente, dal metabolismo delle proteine ingerite e così una riduzione della generazione di queste sostanze nell'organismo, ottenuta restringendo l'apporto di proteine con la dieta, costituisce il razionale della terapia conservativa dell'insufficienza renale. Poiché la concentrazione plasmatica delle sostanze tossiche è determinata non solo dalla loro produzione, ma anche dalla loro eliminazione, nei pazienti in dialisi è possibile controllare la tossicità uremica modulando non solo la generazione meta- bolica con una dieta appropriata (normoproteica, non propriamente ipoproteica), ma anche l'escrezione dialitica delle sostanze.

Sulla base di queste considerazioni circa la patogenesi della sindrome uremica, l'adeguatezza della terapia dialitica è stata storicamente valutata mediante il monitoraggio della concentrazione plasmatica di alcune sostanze tossiche.

\section{L'urea come "marker" della tossicità uremica}

Sebbene la teoria della tossicità delle "Medie Molecole" rimanga una questione ancora dibattuta (1), vi è sato un favore progressivo nel considerare l'urea quale utile "marker" della tossicità uremica e quindi come molecola di riferimento per il monitoraggio della terapia dialitica. Sia ragioni teoriche, sia motivi pratici supportano questa scelta: l'urea è prodotta in quantità proporzionale al catabolismo delle proteine ed è considerata molecola rappresentativa anche della generazione delle altre molecole tossiche, l'urea è facilmente dosabile ed è una sostanza disciolta in tutta l'acqua corporea che viene eliminata dall'organismo durante la dialisi secondo una cinetica a "pool singolo".
Lo Studio Collaborativo Nazionale sulla Dialisi (NCDS) eseguito negli Stati Uniti è stato il primo studio prospettico sulla tossicità uremica nel quale la dialisi è stata erogata in base alla cinetica dell'urea (2).

L'assunto dello studio era che i risultati clinici dipendessero da una certa concentrazione di tossine (rappresentata da 80 $\mathrm{mg} / \mathrm{dl}$ di azoto ureico) e quindi la dialisi era modulata per ottenere concentrazioni ematiche inferiori o superiori al valore prefissato. In tale assunto era anche insito il concetto che gli effetti tossici in una certa concentrazione di molecole fossero identici sia quando causati dalla produzione metabolica, sia quando causati dalla eliminazione dialitica delle sostanze. Considerando che la dialisi era somministrata in funzione dei livelli di BUN, tutti i pazienti con bassa produzione di urea (e di conseguenza con bassi livelli di BUN) ricevevano una bassa quantità di dialisi, sia se appartenenvano al gruppo con BUN inferiore a $80 \mathrm{mg} / \mathrm{dl}$ e ancor più se appartenevano al gruppo con BUN superiore. Quindi la terapia dialitica era erogata non solo in funzione del valore prefissato di BUN, ma anche della quantità di proteine catabolizzate dai pazienti (nP$\mathrm{CR}$ ) e queste due variabili (inoltre intercorrelate) costituivano le variabili di controllo della terapia. La depurazione dialitica (clearance) dell'urea era rilevante in 
questo contesto solo perché contribuiva a modificare i livelli ematici di urea e non perché dovesse essere erogata secondo una certa quantità stabilita a priori.

Alla conclusione dello studio NCDS sia il valore di BUN sia il valore di nPCR si rivelarono predittivi dei risultati clinici dei pazienti: infatti fu trovata una stretta associazione tra valori elevati di BUN e valori bassi di nPCR (inferiori a $0.8 \mathrm{~g} / \mathrm{kg}$ ) con una maggior mortalità e morbilità (3). Due anni più tardi, tuttavia, l' "analisi meccanicistica" dei risultati dell'NCDS fatta da Sargent e Gotch mostrava che la probabilità di avere cattivi risultati clinici dipendeva dalla dose di dialisi somministrata $(\mathrm{Kt} / \mathrm{V})$ in aggiunta o al posto dei livelli plasmatici di azoto ureico e dei valori di nPCR (4). Il ruolo sia delle sostanze tossiche sia dell'introito dietetico di proteine (identificato dall'nPCR) non potevano essere stabiliti separatamente uno dall'altro, poiché il disegno stesso dello studio e il modello cinetico dell'urea davano luogo ad una stretta associazione di BUN ed nPCR tra loro e con il Kt/V erogato.

\section{$\mathrm{Kt} / \mathrm{V}$ e adeguatezza dialitica}

Molti studi hanno successivamente confermato che $\mathrm{Kt} / \mathrm{V}$ è un indice utile per eseguire la prescrizione e il monitoraggio della terapia dialitica: i risultati clinici effettivamente migliorano o peggiorano in stretta associazione con i valori più alti 0 più bassi di Kt/V (5-7).

E importante notare che lo studio NCDS era stato concepito sull'ipotesi dell'esistenza di una correlazione tra la probabilità di sopravvivenza o il numero dei ricoveri e i livelli plasmatici di molecole tossiche, mentre non fu possibile dimostrare una sicura relazione di causa ed effetto tra BUN e risultati clinici.

Ciò non significa che l'assunto del ruolo patogenetico delle sostanze tossiche di piccolo peso molecolare non fosse corretto, dal momento che la probabilità di insuccesso terapeutico anche se non direttamente correlata ai livelli di BUN risulta direttamente dipendente dalla clearance relativa dell'urea $(\mathrm{Kt} / \mathrm{V})$.

Sulla base dei risultati dell'NCDS e di molti studi successivi il trattamento dialitico viene ora considerato adeguato quando la clearance dell'urea erogata durante ogni dialisi equivale alla grandezza dell'acqua del corpo del paziente, cioè quando $\mathrm{Kt} / \mathrm{V}$ è uguale ad 1 .

Di conseguenza e contrariamente all'ipotesi originale dello studio NCDS, la clearance dialitica dell'urea non deve essere erogata in funzione dei valori di BUN e di nPCR, ma deve essere comunque erogata in quantità pari alla grandezza dell'acqua del corpo del paziente.

Nello studio NCDS in cui la clearance dell'urea veniva stabilita in funzione dei valori di BUN prefissati, tutti i pazienti che spontaneamente presentavano bassi valori di BUN (perché introducevano poche proteine e producevano poca urea) ricevevano una quantità di terapia dialitica insufficiente (Kt/V inferiore a 0.8$)$.

Mentre ora è chiaro che la terapia dialitica non deve essere ridotta sotto il limite di sicurezza $(\mathrm{Kt} / \mathrm{V}=1)$ quando i valori di nPCR e di BUN sono spontaneamente bassi, non è conosciuto fino a quale punto possono essere tollerati elevati valori di BUN dipendenti da un eccessivo introito di proteine, ma accompagnati da un $\mathrm{Kt} / \mathrm{V}$ adeguato. Nei nostri pazienti dializzati 4 h 3 volte la settimana abbiamo dimostrato una stretta correlazione tra i valori predialitici di BUN misurati a metà della settimana e i valori di nPCR stimati con la misura dell'urea nel dializzato (8). L'analisi statistica mostrava che valori di BUN pari a $80 \mathrm{mg} / \mathrm{dl}$, cioè il limite superiore secondo le linee guida dello studio NCDS, corrispondevano a valori medi di nPCR di $1.17 \mathrm{~g} / \mathrm{kg}$ di peso corporeo.

Questi risultati venivano ottenuti in pazienti con un peso corporeo medio di 62 $\mathrm{kg}$, dializzati con una clearance media dell'urea in vitro di $190 \mathrm{ml} / \mathrm{min}$ e un $\mathrm{Kt} / \mathrm{V}$ corrispondente a 1.3. Indipendentemente dal fatto che elevati livelli di BUN siano o non siano tossici in presenza di una adeguata dose dialitica, si deve ricordare che, ove non si attui alcuna limitazione dietetica, possono presentarsi alterazioni dell'equilibrio acido-base, iperpotassiemia, cattivo controllo dei fluidi corporei ed iperfosforemia che può causare un peggioramente dell'osteodistrofia.

\section{nPCR e risultati clinici}

Un'evidenza chiara della presenza di malnutrizione proteica può essere trovata nella maggior parte degli studi sui pazienti in dialisi e una sindrome di grave malnutrizione risulta essere presente nel 10-15\% dei pazienti (9). La malnutrizione rappresenta un fattor prognostico negativo per i risultati clinici a lungo termine ed è stata infatti trovata un'associazione tra un basso introito proteico $(10,11)$ o basse albuminemie $(11,12)$ e un aumento delle ospedalizzazioni e della mortalità. È ora ben conosciuto che la quantità minima di proteine necessaria giornalmente per un soggetto sano è circa $0.6 \mathrm{~g} / \mathrm{kg}$ di peso corporeo e che un simile introito di proteine è sufficiente per mantenere in pareggio il bilancio azotato in soggetti uremici non dializzati (13). Al contrario, in alcuni pazienti dializzati può essere trovata un'evidenza di malnutrizione anche con un introito di proteine di $1 \mathrm{~g} / \mathrm{kg}$ (14) ed è necessaria una quantità maggiore di proteine per mantenere il pareggio del bilancio azotato (15).

Sulla base di questi dati, l'introito proteico dei pazienti deve essere regolarmente controllato mediante interviste o diari dietetici, sebbene la determinazione dell'nPCR con la cinetica dialitica dell'urea sia largamente utilizzata quale stima indiretta del consumo dietetico di proteine.

\section{nPCR come stima dell'introito di proteine}

Per poter applicare questo metodo devono essere soddisfatte diverse condizioni, la più importante delle quali è rappresentata dalla stabilità del metabolismo azotato dei pazienti. Nei soggetti con bilancio azotato in pareggio la quantità di proteine catabolizzate (nPCR) equivale infatti alla quantità di proteine introdotte con l'alimentazione. Tuttavia, l'esistenza di uno stato metabolico stabile è desunta dalla cosiddetta stabilità clinica del paziente, ma il concetto di stabilità clinica è tutt'altro che ben definito.

Noi abbiamo studiato 27 pazienti non diabetici, 8 femmine e 19 maschi, in trattamento dialitico da 11 a 75 mesi (16). Questi pazienti erano stati scelti sulla base di due criteri principali: 1) una condizione clinica stabile, cioè assenza di recenti variazioni del peso corporeo, di infezioni, di alterazioni del tratto gastrointestinale e di malattie sistemiche; 2) la capacità di fornire informazioni accurate sull'introito alimentare. 
Tutti i pazienti erano addestrati dallo staff medico e dalla dietista in un programma di sorveglianza nutrizionale periodica. $\mathrm{Ai}$ pazienti veniva fornita una bilancia dietetica e veniva richiesto di pesare i cibi prima della cottura. Quando necessario la precisione nello stimare le quantità di cibo assunto veniva aumentata con la pesata dei piatti prima e dopo il pasto.

Sebbene il valore medio di nPCR risultasse molto prossimo a quello dell'introito di proteine, l'nPCR era più alto dell'introito di proteine nei pazienti con il più basso apporto dietetico di proteine. Valori di nPCR più alti dell'introito dietetico di proteine sono stati trovati in altri studi e sono stati attribuiti sia ad una sottostima della quantità di cibo introdotta, sia ad un ipercatabolismo proteico.

Nel nostro studio abbiamo osservato che i valori di nPCR erano superiori alla quantità di proteine introdotte solo nei pazienti che avevano il più basso apporto di proteine e questa categoria di pazienti è effettivamente a rischio di sviluppare un bilancio azotato negativo, cioè una produzione di azoto ureico in eccesso derivante dal catabolismo delle proteine proprie dell'organismo. Ed infatti, il bilancio azotato, stimato dal rapporto tra l'urea nitrogen appearance (azoto ureico prodotto) e l'azoto introdotto con le proteine della dieta, risultava essere negativo in sette pazienti su dodici che avevano una introduzione di proteine inferiore ad $1 \mathrm{~g} / \mathrm{kg}$ di peso corporeo e il bilancio azotato era neutro o positivo in tredici su quindici pazienti che avevano una introduzione di proteine superiore a $1 \mathrm{~g} / \mathrm{kg}$ di peso corporeo.

Poiché i nostri pazienti erao stati scelti sulla base di una loro condizione clinica stabile, questi risultati dimostrano che il concetto corrente di stabilità clinica non implica regolarmente l'esistenza di una condizione di stabilità metabolica.

Nel nostro studio il 25\% dei pazienti non erano a bilancio azotato neutro, ma presentavano uno stato più o meno accentuato di ipercatabolismo proteico o, più raramente, di anabolismo proteico. Secondo i nostri dati, quando si trovino valori di $\mathrm{nP}$ CR superiori a $1 \mathrm{~g} / \mathrm{kg}$ è molto probabile che essi rappresentino un indice attendibile della quantità di proteine introdotte, mentre valori di nPCR inferiori a 1 possano soprastimare (bilancio azotato negativo) e più raramente sottostimare la reale introduzione di proteine (bilancio azotato positivo).
Quando i valori di nPCR siano inferiori a $1 \mathrm{~g} / \mathrm{kg}$ è quindi necessario fare anche una valutazione diretta dell'introito dietetico sia di proteine che di calorie in modo da stimare il reale introito proteico e l'entità dello scostamento della nutrizione dai valori ideali.

\section{Fattori che influenzano nPCR}

Come detto in precedenza, alcuni pazienti possono essere catabolici ed altri anabolici, ma nella maggioranza vi è il pareggio azotato e quindi i valori di nPCR sono generalmente proporzionali alla quantità di proteine introdotte giornalmente. Diversi fattori possono influenzare l'introito proteico nei pazienti in dialisi e di conseguenza i valori di nPCR, ma recentemente sono emersi due nuovi fattori: il tipo dei filtri utilizzati e la dose dialitica somministrata. Infatti, Lindasy et al (17) hanno postulato che un aumento della terapia dialitica rappresentato da un aumento del $\mathrm{Kt} / \mathrm{V}$ e l'uso di membrane più permeabili alle molecole tossiche potrebbero favorire l'appetito dei pazienti e aumentare l'introito di proteine (e quindi l'nPCR). Dal momento che è stata trovata una correlazione diretta tra $\mathrm{Kt} / \mathrm{V}$ ed nPCR e tale correlazione è lineare dai livelli bassi ai livelli più elevati di efficienza dialitica $(\mathrm{Kt} / \mathrm{V})$, questi Autori hanno suggerito che un aumento della dose dialitica sia un provvedimento da prendere per stimolare l'introito di proteine nei pazienti che non presentino cause ovvie di malnutrizione e nei quali i valori di nPCR siano inferiori a $1 \mathrm{~g} / \mathrm{kg}$ (18). Questa teoria è in contrasto con i risultati dello studio NCDS in cui una più alta probabilità di insuccesso della terapia era effettivamente associata con valori bassi di $\mathrm{Kt} / \mathrm{V}$ (inferiori a 0.8 ), ma nel quale i risultati clinici non miglioravano progressivamente quando i valori di $\mathrm{Kt} / \mathrm{V}$ superavano 0.8 e crescevano da 0.9 fino a 1.5 (4).

Il significato da attribuire alla correlazione tra Kt/V ed nPCR è incerto. È stato sottolineato che questa correlazione potrebbe anche essere frutto di una semplice tautologia matematica dal momento che $i$ valori di $\mathrm{Kt} / \mathrm{V}$ e nPCR derivano da fattori comuni (19-21). Si dubita cioè che l'esistenza di una relazione diretta tra i due parametri significhi necessariamente un interdipendenza tra la quantità di dialisi ricevuta e l'alimentazione del paziente, particolarmente quando la dose dialitica sia superiore a quella riteuta adeguata $(\mathrm{Kt} / \mathrm{V}$ uguale a 1$)$. Infatti, mentre è evidente che una quantità di dialisi insufficiente può comportare la perdita dell'appetito e quindi un basso introito di proteine (e un basso nPCR) e che una più efficiente rimozione dei tossici uremici può stimolare l'appetito, è molto più discutibile che quantità di dialisi ed introito di proteine abbiano una relazione di causa ed effetto quando la dose dialitica somministrata sia già appropriata $(\mathrm{Kt} / \mathrm{V}$ maggiore di 0.8 ). Per chiarire questa questione sarebbe necessario uno studio prospettico sottoponendo gli stessi pazienti a dialisi con diversa efficienza dialitica, però sempre sopra il limite adeguato, ma uno studio simile non è ancora stato realizzato. In uno studio precedente abbiamo valutato il metabolismo proteico in un gruppo di pazienti caratterizzati dall'essere ben dializzati (22). Furono valutati i valori di nPCR e contemporaneamente l'introito dietetico di proteine in modo da correlare la dose dialitica $(\mathrm{Kt} / \mathrm{V})$ non solo con l'nP$\mathrm{CR}$ ma anche direttamente con le proteine introdotte; in questo modo è stato anche possibile escludere gli effetti di una dialisi non adeguata sull'alimentazione. In questo studio non abbiamo potuto trovare alcuna relazione tra dose dialitica ed introito di proteine; è anche interessante notare che l'nPCR non era correlato con il $\mathrm{Kt} / \mathrm{V}$ e ciò suggerisce che, qualunque sia il significato (matematico? fisiologico?) della relazione tra $\mathrm{Kt} / \mathrm{V}$ e $\mathrm{nPCR}$, la relazione non sia più tanto stretta quando $\mathrm{i}$ valori di $\mathrm{Kt} / \mathrm{V}$ siano superiori a 0.8 . Questi dati sono in accordo con i risultati di due studi recenti che fanno riferimento a pazienti ben diabilizzati, nei quali non fu trovata nessuna correlazione tra valori di $\mathrm{Kt} / \mathrm{V}$ e nPCR $(23,24)$.

Secondo questi dati la nutrizione appare dialisi-indipendente o meglio una variabile paziente-dipendente quando l'efficienza del trattamento sia garantita (25). Tra gli indici studiati l'età dei pazienti risultava inversamente correlata con l'introito di proteine e ciò suggerisce che i pazienti anziani possono sviluppare con maggior facilità una malnutrizione. L'introito di proteine più che la dose dialitica risultava il fattore critico nel determinare il bilancio azotato che era negativo quando l'introito di proteine era inferiore a $0.94 \mathrm{~g} / \mathrm{kg}$ di peso corporeo. 


\section{BIBLIOGRAFIA}

1. Vanholder R, De Smet R, Hsu C, Vogeleere P, Ringoir S. Uremic toxicity: the middle molecule hypothesis revisited. Seminars in Nephrology 1994; 14: 205-18.

2. Lowrie EG, Laird NM, Henry RR. Protocol for the National Cooperative Dialysis Study. Kidney Int 1983; 23 (suppl 13): 11-8.

3. Parker TF, Laird NM, Lowrie EG. Comparison of the study groups in the National Cooperative Dialysis Study and a description of morbidity, mortality, and patient withdrawal. Kidney Int 1983; 23 (suppl 13): 42-9.

4. Gotch FA, Sargent A. A mechanistic analysis of the National Cooperative Dialysis Study (NCDS). Kidney Int 1985; 28: 52634.

5. Hakim RM. Assessing the adequacy of dialysis. Kidney Int 1990; 37: 822-32.

6. Acchiardo SR. Uremia and adequate dialysis treatment. Seminars in Nephrology 1994; 14: 27481.

7. Hakim RM, Breyer J, Ismail N, Schulman G. Effects of dose of dialysis on morbidity and mortality. Am J Kidney Dis 1994; 23: 661-9.

8. Panzetta G, Maschio G. Dietary problems of the dialysis patient. Blood Purif 1985; 3: 63-74.

9. Feinstain EI, Kopple JD. Severe wasting and malnutrition in a patient undergoing maintanance dialysis. Am J Nephrol 1985; 5: 398-405.

10. Acchiardo S, Moor L, Latour P. Malnutrition as the main factor in morbidity and mortality of hemodialysis patients. Kidney Int 1983; 24 (suppl 16): S199-203.
11. Lowrie EG, Lew N. Death risk in hemodialysis patients. The predictive value of commonly measured variables and an evaluation of death rate differences between facilities. Am J Kidney Dis 1990; 15: 458-82.

12. Goldwasser P, Mittman N, Antigani A, et al. Predictors of mortality in hemodialysis patients. J Am Soc Nephrol 1993; 3: 1613-22.

13. Monteon JF, Laidlaw SA, Shaib JK, Kopple JD. Energy expenditure in patients with chronic renal failure. Kidney Int 1986; 30: 741-7.

14. Blumenkrantz MJ, Kopple JD, Gutman RA, et al. Methods for assessing nutritional status of patients with renal failure. Am J Clin Nutr 1980; 33: 1567-85.

15. Borah MF, Schoenfeld PY, Gotch FA, Sargent JA, Wolfson M, Humphreys MH. Nitrogen balance during intermittent dialysis therapy of uremia. Kidney Int 1978; 14: 4491-500.

16. Panzetta G, Tessitore N, Faccini G, Maschio G. The protein catabolic rate as a measure of protein intake in dialysis patients: usefulness and limits. Nephrol Dial Transplant 1990; suppl. 1: 125-7.

17. Lindsay RM, Spanner E. A hypothesis: the protein catabolic rate is dependent upon the type and amount of treatment in dialyzed uremic patients. Am J Kidney Dis 1989; 13: 382-9.

18. Lidnsay RM, Spanner E. Heidenheim P, et al. Which comes first, $\mathrm{Kt} / \mathrm{V}$ or PCR-chicken or egg? Kidney Int 1992; 42 (suppl 38): S32-6.

19. Bergstrom J, Furst P, Alvestrand A, et al. Protein and energy intake, nitrogen balance and nitrogen losses in patients treated with continuous ambulatory peritoneal dialysis. Kidney Int 1993; 44: 104857.

20. Stein A, Walls J. The correlation between $\mathrm{Kt} / \mathrm{V}$ and protein catabolic rate a self-fulfilling prophecy.
Nephrol Dial Transplant 1994; 9 : 743-5.

21. Venning MG, Faragher EB Harty JC, et al. The relationship between $\mathrm{Kt} / \mathrm{V}$ and $\mathrm{nPCR}$ in hemodialysis patients in cross-sectional studies in mathematical coupling. J Am Soc Nephrol 1993; 4: 393 (abstract).

22. Panzetta G, Bianco F, Tessitore N, et al. Factors affecting nitrogen balance in hemodialysis patients. Control Nephrol 1992; 98: 183-91.

23. Movilli E, Gaggiotti M, Sandrini M, et al. Effect of age on protein catabolic rate, morbidity and mortality in uremic patients with adequate normalized dose of dialysis. Nephrol Dial Transplant 1992; 66: 438-41.

24. Morgenstre A, Winkler J, Narkis R, et al. Adequancy of dialysis and nutrition status in hemodialysis patients. Nephro 1994; 66: 428-41.

25. Panzetta G. Protein intake does not depend on the dose of dialysis delivered- provided $\mathrm{Kt} / \mathrm{V}$ is adequate. N.D.T. 1996; 10: 2286-9. 